\title{
A NOTE ON THE CATEGORY OF THE FREE LOOP SPACE
}

\author{
E. FADELL AND S. HUSSEINI
}

(Communicated by Fredrick R. Cohen)

\begin{abstract}
A useful result in critical point theory is that the LjusternikSchnirelmann category of the space of based loops on a compact simply connected manifold $M$ is infinite (because the cup length of $M$ is infinite). However, the space of free loops on $M$ may have trivial products. This note shows that, nevertheless, the space of the free loops also has infinite category.
\end{abstract}

\section{INTRODUCTION}

It is a standard result that if $M$ is a simply connected compact manifold and $\Omega(M)=\Omega\left(M, x_{0}\right)$ is the space of based loops on $M$ (based at $\left.x_{0}\right)$, then the Ljusternik-Schnirelmann category cat $\Omega(M)=+\infty$. This follows from the now classical result (Serre [11], that the real (or rational) cohomology of $\Omega(M)$ has nontrivial cup products of arbitrary high length (see also [10]). An inspection of the proof will convince the reader that compactness is not required for the proof of this result. All that is required is that the real (or rational) cohomology $H^{*}(M)$ be finitely generated and for some $i>0 H^{i}(M) \neq 0$. However, for the free loop space $\Lambda(M)$, where

$$
\Lambda(M)=\left\{\alpha \in M^{I}, \alpha(0)=\alpha(1)\right\},
$$

it isn't necessarily the case that the cohomology of $\Lambda(M)$ has nontrivial cup products. This is a relatively recent result of $M$. Vigué-Poirrier and D. Sullivan [13], where, for example, the reduced real cohomology of the free loops on the 2-sphere $S^{2}$ has trivial cup products. In view of this fact, it is natural to inquire about that category of the free loop space $\Lambda(M)$. We will show that when $M$ satisfies the preceding conditions,

1. $\operatorname{cat} \Lambda(M)=+\infty$

2. $\Lambda(M)$ contains compact subsets $C$ such that $\operatorname{cat}_{\Lambda(M)} C$ is arbitrarily large.

Received by the editors May 18, 1988, and in revised form, January 18, 1989.

1980 Mathematics Subject Classification (1985 Revision). Primary 58E05, 55P35, 55R20.

Supported in part by NSF Grant DMS-8722295.. 
This will allow a direct application of the Ljusternik-Schnirelmann method to, for example, functionals defined on the Sobolev space

$$
W_{T}^{1,2}=\left\{f:[0, T] \rightarrow \mathbf{R}^{n}-0, f(0)=f(T)\right\} .
$$

See $\S 4$ and [1], [9].

The basic result is the following property of Hurewicz fibrations [7], which we prove in the next section.

Theorem. Let $F \stackrel{i}{\longrightarrow} E \stackrel{p}{\longrightarrow} B$ denote a Hurewicz fibration which admits a section $\sigma: B \rightarrow E$, and $F, B$, and $E$ are 0-connected. If $Q \subset F$ is any subset of $F$, then

$$
\operatorname{cat}_{F} Q \leq \operatorname{cat}_{E} Q \text {. }
$$

When applied to the fibration $\Omega(M) \rightarrow \Lambda(M) \rightarrow M$ we obtain:

Corollary. Let $M$ denote a simply connected manifold (not necessarily compact) such that the real or rational cohomology $H^{q}(M)$ is finitely generated for each $q$ and $H^{i}(M) \neq 0$ for some $i>0$. Then cat $\Lambda(M)=\infty$.

$\S 3$ considers the category of the free loop space on configuration spaces.

\section{RESUlts}

We recall first a basic lemma for Hurewicz fibrations [2]. If $F \stackrel{i}{\longrightarrow} E \stackrel{p}{\longrightarrow} B$ is a Hurewicz fibration, then there is a lifting function $\lambda: \Omega_{p} \rightarrow E^{I}$, where $\Omega_{p}=\left\{(x, \omega) \in E \times B^{I}: p(x)=\omega(0)\right\}$, where $\lambda(x, \omega)(0)=x ; p(\lambda(x, \omega)(t))=$ $\omega(t), 0 \leq t \leq 1 . \lambda$ induces $\tilde{\lambda}: E^{I} \rightarrow E^{I}$ by setting $\tilde{\lambda}(\alpha)=\lambda[\alpha(0), p \alpha]$.

2.1. Lemma [7]. $\tilde{\lambda} \sim \mathrm{id}$ preserving projections, i.e., there is a homotopy $\Gamma$ : $E^{I} \times I \rightarrow I$ such that $\Gamma_{0}=\mathrm{id}, \Gamma_{1}=\tilde{\lambda}$ and $p \Gamma(\alpha, s)(t)=p \alpha(t)$ for $\alpha \in E^{I}$, $s, t \in I$.

2.2. Proposition. Let $F \stackrel{i}{\longrightarrow} E \stackrel{p}{\longrightarrow} B$ denote a Hurewicz fibration with base points $x_{0} \in F, b_{0} \in B, F=p^{-1}\left(b_{0}\right)$. We assume that $\Omega_{p}: \Omega\left(E, x_{0}\right) \rightarrow$ $\Omega\left(B, b_{0}\right)$ admits a section $\sigma$. If $Y$ is any space and $f: Y \rightarrow F$ is a map homotopic in $E$ to the constant $x_{0}$, the $f$ is homotopic in $F$ to $x_{0}$.

Proof. Let $A: Y \rightarrow E^{I}$ denote a homotopy such that $A(y)(0)=x_{0}, A(y)(1)=$ $f(y)$. Consider the homotopy $\widehat{A}: Y \times I \rightarrow F$ given by

$$
\widehat{A}(y, s)=\Gamma(A(y), s)(1), \quad 0 \leq s \leq 1, \quad y \in F .
$$

Note that $\widehat{A}(y, 0)=f(y), \widehat{A}(y, s) \in F$ for $0 \leq s \leq 1$. Let $\widehat{A}(y, 1)=g(y)$ so that $f \sim g: Y \rightarrow F$.

Now, define $C: Y \rightarrow E^{I}$ by $C(y)=\sigma p A(y)$ and observe that $p C(y)=$ $p A(y)$. Now, define a homotopy $\widehat{C}: Y \times I \rightarrow F$ by

$$
\widehat{C}(y, s)=\Gamma(C(y), s)(1), \quad 0 \leq s \leq 1, \quad y \in F .
$$


Note that $\widehat{C}(y, 0)=x_{0}, \widehat{C}(y, 1)=g(y)$, and $\widehat{C}(y, s) \in F, 0 \leq s \leq 1$. Thus, $f \sim x_{0}: Y \rightarrow F$ and the proposition follows.

2.3. Remark. The reader will note the similarity between Proposition 2.2 and the classical result that $i_{*}: \pi_{*}(F) \rightarrow \pi_{*}(E)$ is injective when $p$ admits a section [12]. Proposition 2.2 may also be thought of as a situation when the fiber is totally non-homotopic to zero.

Now we recall that a set $Q$ in $Y$ is categorical in $Y$ if the inclusion $i: Q \longrightarrow$ $Y$ is homotopic in $Y$ to the constant map $y_{0} \in Y$

2.4. Corollary. Let $F \stackrel{i}{\longrightarrow} E \stackrel{p}{\longrightarrow} B$ denote a Hurewicz fibration as in 2.2 with $E$ 0-connected. If $U$ is categorical in $E$, then $U \cap F$ is categorical in $F$. Consequently, for any subset $Q \subset F$,

$$
\operatorname{cat}_{F} Q \leq \operatorname{cat}_{E} Q .
$$

Proof. The first part follows from Proposition 2.2, while the second part is immediate from the definition of category, namely, cat $_{Y} X$ is the minimum number of categorical open sets in $Y$ which cover $X$.

2.5. Definition. A manifold $M$ will be called admissible if $M$ is simply connected, the real (or rational) cohomology $H^{*}(M)$ is finitely generated and for some $i>0, H^{i}(M) \neq 0$.

2.6. Corollary. Let $M$ denote a simply connected manifold with base point $x_{0}$. Then, we have the fibration with section

$$
\Omega\left(M, x_{0}\right) \stackrel{i}{\longrightarrow} \Lambda(M) \stackrel{p}{\stackrel{p}{\rightleftarrows}} M
$$

where $p(\omega)=\omega(0)$ and $\sigma(x)=\tilde{x}$ the constant loop at $x \in M$. If $Q \subset$ $\Omega\left(M, x_{0}\right)$ is an subset

$$
\operatorname{cat}_{\Omega\left(M, x_{0}\right)} Q \leq \operatorname{cat}_{\Lambda(M)} Q .
$$

In particular, if $M$ is admissible, then cat $\Lambda(M)=\infty$.

2.7. Remark. Thus for example $\Lambda\left(S^{2}\right)$ has infinite category but trivial cup products over R [13].

2.8. Remark. Suppose $F$ is a closed subset of $E$ and $Q \subset F$. Then, the reverse inequality $\mathrm{cat}_{E} Q \leq \mathrm{cat}_{F} Q$ holds whenever $E$ is and $\mathrm{ANR}$ (normal). This is the case in Corollary 2.4 and hence there the inequality is actually an equality.

Now, we consider the question of compact subsets of $\Lambda(M)$ of arbitrarily high category.

2.9. Lemma. Suppose $X$ is a space such that for some field $F$ the cup length of $X$ over $\mathbf{F}$ using singular cohomology is $\geq k$; then $X$ has a compact subset of category $>k$.

Proof. We mention first that throughout we employ singular homology and cohomology with coefficients in $F$ and will make use of the universal coefficient 
theorem isomorphism

$$
\gamma: H^{q}(X) \longrightarrow \operatorname{Hom}_{\mathbf{F}}\left(H_{q}(X) ; \mathbf{F}\right) .
$$

Let $w=\alpha_{1} \alpha_{2} \cdots \alpha_{k} \in H^{q}(X)$ denote a nontrivial cup product of length $k$. Then, $\gamma(w) \neq 0$ and hence there is a singular cycle $\zeta$ such that $\gamma(w)([\zeta]) \neq 0$. Let $A$ denote the (compact) support of $\zeta$. Then, it is easy to check that if $i: A \rightarrow X$ is the inclusion map, $i^{*}(w)=i^{*}\left(\alpha_{1}\right) \cdots i^{*}\left(\alpha_{k}\right)$ is nonzero in $H^{q}(A)$. Thus the cup length of $A$ in $X$ is $\geq k$ and cat $_{X} A>k$.

2.10. Corollary. Let $M$ denote an admissible manifold with base point $x_{0}$. Then, the space of based loops $\Omega\left(M, x_{0}\right)$ (and hence the space of free loops $\Lambda(M))$ contains compact subsets of arbitrarily high category.

\section{CONFiguration SPACES}

If $M$ is any space the $k$ th configuration space of $X, k \geq 1$, is defined by (see [6])

$$
F_{k}(M)=\left\{\left(x_{1}, \ldots, x_{k}\right), x_{i} \in M, x_{i} \neq x_{j}, \quad \text { for } i \neq j\right\} .
$$

We will make of the following propositions. Cohomology will be over a field $F$ of coefficients.

3.1. If $M$ is a manifold (without boundary) and $k \geq 2$, then we have locally trivial fibrations

$$
F_{k-1}(M-Q) \rightarrow F_{k}(M) \stackrel{p}{\rightarrow} M
$$

where $Q \in M$ and $p\left(x_{1}, \ldots, x_{k}\right)=x_{k}$; and

$$
\left(M-Q_{k-1}\right) \rightarrow F_{k}(M) \stackrel{q}{\rightarrow} F_{k-1}(M)
$$

where $Q_{k-1} \subset M$ is a subset of $k-1$ elements and $q\left(x_{1}, \ldots, x_{k}\right)=$ $\left(x_{1}, \ldots, x_{k-1}\right)$.

3.2. If $M$ is a simply connected manifold, $\operatorname{dim} M=m \geq 3$, and $H^{i}(M)$ is finitely generated over a field $\mathbf{F}$ for each $i$, then for $k \geq 1 F_{k}(M)$ is simply connected and $H^{i}\left(F_{k}(M)\right)$ and $H^{i}\left(\Omega F_{k}(M)\right)$ are finitely generated over $\mathbf{F}$ for each $i$.

We prove the proposition.

3.3. Proposition. If $M$ is a simply connected manifold, $\operatorname{dim} M=m \geq 3$, then for $k \geq 2$, the configuration space $F_{k}(M)$ is admissible.

Proof. Because of 3.2 and the fact that $F_{k}(M)$ is finite dimensional, we need only show that for some $j>0$, the real cohomology $H^{j}\left(F_{k}(M)\right) \neq 0$.

Case 1. $H^{i}(M) \neq 0$ for some $i \geq 1$. Choose $i$ maximal so that $H^{i}(M) \neq$ 0 and $0 \neq v \in H^{i}(M)$. We proceed by induction on $k$ and employ the 
cohomology spectral sequence of the fibration (i) of 3.1. Choose $u \in F_{k-1}(M-$ $Q)$ of maximal dimension so that $u \neq 0$. Then in the $E_{2}$-term of the spectral sequence $u \otimes v \neq 0$ and has dimension $>0$. It is easy to see that $u \otimes v$ "survives" to $E_{\infty}$ and contributes a nonzero element to $H^{j}\left(F_{k}(M)\right), j \geq 1$.

Case 2. $H^{i}(M)=0$ for all $i>0$. For $k=2$ we employ the spectral sequence of the fibration (i) of 3.1 to see that $\mathrm{R}=H^{m-1}(M-Q) \approx H^{m-1}\left(F_{2}(M)\right)$. For $k \geq 3$, we employ induction on $k$ and the spectral sequence of the fibration (ii) of 3.1, together with the argument in Case 1 to obtain the desired result.

3.4. Proposition. If $M$ is a simply connected manifold, $\operatorname{dim} M \geq 3$, then for $k \geq 2$, cat $\Lambda F_{k}(M)=\infty$ and $\Lambda F_{k}(M)$ contains compact subsets of arbitrarily high category.

\section{AN APPLication}

In [9], Rabinowitz used the main result of $\S 2$ (corollary 2.10) in the special case where $M=\mathbf{R}^{M}-\{0\}$ to prove the existence of infinitely many periodic solutions of a certain Hamiltonian system. In this section we give an alternative argument for a key proposition in his treatment based upon a general abstract critical point theorem. That theorem is the analogue of a previous "linking" result in [5] done in the context of a relative cohomological equivariant index theory which will be replaced here by relative (Ljusternik-Schnirelmann) category theory introduced in [3] and [4].

We review first one version of relative category. If $(E, A)$ is a topological pair with $A \neq \phi$ and closed in $E$, then for $A \subset X \subset E$ we define the relative category $\operatorname{cat}_{E}(X, A)$, as follows. A categorical cover of $(X, A)$ consists of an open (in $E$ ) set $W \supset A$ and open sets $\left\{V_{j}\right\}$ such that

1. $W \cup\left(\cup V_{j}\right) \supset X$.

2. There is a homotopy of pairs $H:(W, A) \times I \rightarrow(E, A)$ such that $H_{0}(x)=x$ and $H_{1}(x) \in A, x \in A$.

3. Each $V_{j}$ is contractible to a point in $E$.

4.1. Definition. Cat $_{E}(X, A)=n$ if $(X, A)$ admits a categorical cover $\left\{W, V_{j}\right\}$ with $n$ sets $V_{j}$ and $n$ is minimal with this property. If no such finite categorical cover exists we set $\operatorname{cat}_{E}(X, A)=\infty$.

4.2. Remark. If $A=\phi$, $^{2}{ }_{E}(X, \phi)=\operatorname{cat}_{E} X$ has its usual meaning. The following properties are immediate:

\subsection{Proposition.}

(a) $A \subset X_{1} \subset X_{2}$ implies cat ${ }_{E}\left(X_{1}, A\right) \leq \operatorname{cat}_{E}\left(X_{2}, A\right)$.

(b) $A \subset X_{1}, X_{2} \subset E$ implies $\mathrm{cat}_{E}\left(X_{1} \cup X_{2}, A\right) \leq \mathrm{cat}_{E}\left(X_{1}, A\right)+\operatorname{cat}_{E} X_{2}$.

Relative category may be used to define a "linking" concept as follows. 
4.4. Definition. Let $A$ and $B$ denote disjoint closed sets in a space $E$. If

$$
\operatorname{cat}_{E}(E-B, A)<\operatorname{cat}_{E}(E, A)
$$

we say that $A$ and $B \operatorname{link}$ (in the category sense). If, in addition, cat $_{E}(E, A)=$ $+\infty$, we say that $A$ and $B$ strongly link.

We review next a local form of the Palais-Smale condition (PS) $)_{S}$. Let $\Lambda$ denote an open set in a Banach space and $f: \Lambda \rightarrow \mathbf{R}$ a $C^{1}$-functional. $f$ is said to satisfy (PS) if any sequence $q_{j} \in \Lambda$ satisfying $f\left(q_{j}\right) \rightarrow s$ and $f\left(q_{j}\right) \rightarrow 0$ is precompact. (PS $)_{s}$ is used crucially in the following deformation theorem ([8], [9]). We will use that notation $K_{c}=\left\{q \in \Lambda, f^{\prime}(c)=0\right.$, and $\left.f(q)=c\right\}$. Also $f^{a}=\{q \in \Lambda: f(q)<a\}$.

4.5. Proposition. Let $\Lambda$ denote an open set in a Banach space $E$ and $f: \Lambda \rightarrow \mathbf{R}$ a $C^{1}$-functional. Suppose $f$ satisfies (PS) for all $s>\alpha$. Then, for any critical point $c>\alpha, U$ a neighborhood of $K_{c}$, and $\bar{\varepsilon}>0$, there is an $\varepsilon>0$ and $a$ deformation $\varphi: \Lambda \times I \rightarrow \Lambda$ such that

(a) $\varphi_{0}=$ identity, $\varphi_{t}: \Lambda \rightarrow \Lambda$ is a homeomorphism, $t \in[0,1]$.

(b) $\varphi(q, t)=q$ if $|f(q)-c| \geq \bar{\varepsilon}$

(c) $\varphi(q, 1) \in f^{c-\varepsilon}$ if $q \in\left(f^{c+\varepsilon}-U\right)$.

If $K_{c}=\phi$, we may take $U=\phi$.

We may now state our abstract critical point theorem.

4.6. Theorem. Let $\Lambda$ denote an open set in a Hilbert (or Banach) space $E$ such that $\Lambda$ contains compact subsets of arbitrarily large category and let $f: \Lambda \rightarrow \mathbf{R}$ denote a $C^{1}$-functional. Suppose further that there are disjoint closed sets $A$ and $B$ in $\Lambda$ such that

1. cat $_{E}(\Lambda-B, A)<\operatorname{cat}_{E}(\Lambda, A)=\infty$, i.e. $A$ and $B$ strongly link,

2. cat $_{E} A<\infty$,

3. $\sup _{A} f<\inf _{B} f$,

4. $f$ is (PS) $)_{\mathrm{s}}$ for all $s>\sup _{A} f$;

then $f$ possesses an unbounded sequence of critical values.

Proof. For each integer $j \geq 0$ let

$$
\Sigma_{j}=\left\{X \mid A \subset X \subset \Lambda, \operatorname{cat}_{\Lambda}(X, A) \geq j\right\} .
$$

Observe that for each $j$, there is a compact set $Y$ such that cat $Y \geq j+\operatorname{cat}_{E} A$ and hence $\operatorname{cat}_{E}(A \cup Y, A) \geq j$. Thus, $\Sigma_{j}$ is nonempty and $f$ is bounded on $A \cup Y$. Hence, we may define

$$
c_{j}=\inf _{X \in \Sigma_{j}} \sup _{X} f(x), \quad j \geq 0
$$

where

$$
c_{0} \leq c_{1} \leq c_{2} \leq \cdots c_{j} \leq c_{j+1} \leq \cdots \text {. }
$$


Let $m=\operatorname{cat}_{\Lambda}(E-B, A)$. If $\operatorname{cat}_{\Lambda}(X, A) \geq m+1$, then $X \cap B \neq \phi$ for, otherwise $(X, A) \subset(\Lambda-B, B)$ and $\operatorname{cat}_{\Lambda}(X, A) \leq m$. Therefore,

$$
\sup _{A} f=c_{0}<c_{m+1}
$$

i.e. a "jump" occurs at index $m+1$. We now show that each $c_{j}, j>m$, is a critical value. Let $c=c_{j}, j>m$ and consider $K_{c}$. Choose $\bar{\varepsilon}<\frac{1}{2}\left(c_{m+1}-c_{0}\right)$ and an open set $U \supset K_{c}$ such that $U \subset f^{-1}\left(c_{m+1}-\bar{\varepsilon}, \infty\right)$ and cat ${ }_{\Lambda} U=\operatorname{cat}_{\Lambda} K_{c}$. Now let $\varepsilon>0$ and $\varphi$ be the deformation given by Proposition 4.5. Observe that be $\varphi$ remains fixed on $A$ throughout the deformation. Take $X \in \Sigma_{j}$ so that $\operatorname{cat}_{\Lambda}(X, A) \geq j$ and $\sup _{X} f<c+\varepsilon$. Now

$$
\operatorname{cat}_{\Lambda}(X, A) \leq \operatorname{cat}_{\Lambda}(X-U, A)+\operatorname{cat}_{\Lambda}(U) \text {. }
$$

If $K_{c}=\phi$, then $\operatorname{cat}_{\Lambda}(X, A)=\operatorname{cat}_{\Lambda}(X-U, A) \geq j$. On the other hand, $f\left(\varphi_{1}(X-U), A\right)<c-\varepsilon$. Since $\operatorname{cat}_{\Lambda}\left(\varphi_{1}(X-U), A\right)=\operatorname{cat}_{\Lambda}(X-U, A) \geq j$, this would force $c_{j}<c-\varepsilon=c_{j}-\varepsilon$, which is a contradiction. Thus $K_{c} \neq \phi$.

To show that the $c_{j}$ are unbounded we proceed as follows. Let $\bar{c}=\sup c_{j} . \bar{c}$ is again a critical value. Let $K$ denote the set of all critical points $q$ such that $c_{m+1} \leq f(q) \leq \bar{c}$. The (PS) $)_{s}$ condition for all $s>c_{0}$ forces $K$ to be compact and $K \cap A=\phi$. Suppose cat $_{\Lambda} K=k>0$. Again choose $\bar{\varepsilon}<\frac{1}{2}\left(c_{m+1}-c_{0}\right)$ and an open set $U \supset K$ such that $U \subset f^{-1}\left(c_{m+1}-\bar{\varepsilon}, \infty\right)$ and cat $_{\Lambda} U=k$. Furthermore, $\varepsilon$ and $\varphi$ will be as in Proposition 4.5, with $c=\bar{c}$. Choose an index $j$ such that $c_{j}>\bar{c}-\varepsilon$ and $X \in \Sigma_{j+k}$ such that

$$
\sup _{X} f<c_{j+k}+\varepsilon<\bar{c}+\varepsilon \text {. }
$$

Then

$$
\operatorname{cat}_{\Lambda}(X, A) \leq \operatorname{cat}_{\Lambda}(X-U, A)+\operatorname{cat}_{\Lambda}(U)
$$

so that $\operatorname{cat}_{\Lambda}(X-U, A) \geq j$. But, then $\operatorname{cat}_{\Lambda}\left(\varphi_{1}(X-U), A\right) \geq j$ and $\varphi_{1}(X-$ $U, A) \subset f^{\bar{c}-\varepsilon}$. This forces $c_{j}<\bar{c}-\varepsilon$, which is a contradiction.

As an application of Theorem 4.6, we give an alternative proof of a result of P. Rabinowitz, which he used to prove a slightly more general result [9]. The setting is the following Hamiltonian system.

$$
\ddot{q}+V_{q}(t, q)=0
$$

where the potential function $V(t, q)$ satisfies the following conditions:

(V1) $V(t, q)$ is a $C^{1}$-function from $\mathbf{R} \times \Omega \rightarrow \mathbf{R}, \Omega=\mathbf{R}^{n}-\{0\}, n \geq 3$, which is $T$-periodic in $t$.

(V2) $V(t, q)<0$ and $V(t, q) \rightarrow 0, V_{q}(t, q) \rightarrow 0$ as $|q| \rightarrow \infty$, uniformly in $t \in[0, T]$.

(V3) $V(t, q) \rightarrow-\infty$ as $q \rightarrow 0$, uniformly in $t \in[0, T]$.

(V4) There is a neighborhood $N$ of 0 in $\mathbf{R}^{n}$ and a $C^{1}$-function $U: N-$ $\{0\} \rightarrow \mathbf{R}$ such that $U(q) \rightarrow \infty$ as $q \rightarrow 0$ and $-V(t, q) \geq\left|U_{q}(q)\right|^{2}$ for $q \in N-\{0\}$ and all $t \in[0, T]$. 
The period $T>0$ will be fixed throughout the remainder of this section and $E_{T}=W_{T}^{1,2}\left(\mathbf{R}, \mathbf{R}^{n}\right)$ will denote the Sobolev space of $T$-periodic functions with square summable first derivatives, under the norm

$$
\|q\|=\left(\int_{0}^{T}|\dot{q}|^{2} d t+[q]^{2}\right)^{\frac{1}{2}}
$$

where $\dot{q}=\frac{d q}{d t}$ and

$$
[q]=\frac{1}{T} \int_{0}^{T} q(t) d t
$$

Set

$$
\Lambda=\Lambda_{T}\left\{q \in E_{T} \mid q(t) \neq 0 \text { for all } t \in[0, T]\right\} .
$$

$\Lambda$ is an open subset of $E_{T}$ and $\Lambda$ has the same homotopy type as the space $\Lambda\left(\mathbb{R}^{n}-0\right)$ of free loops on $\mathbb{R}^{n}-0 . \mathbb{R}^{n}-0$ is identified with the constant loops in $\mathbf{R}^{n}-0$.

Corresponding to (HS) is the functional $I: \Lambda \rightarrow R$ given by

$$
I(q)=\int_{0}^{T}\left(\frac{1}{2}|\dot{q}|^{2}-V(t, q)\right) d t, \quad q \in \Lambda .
$$

Critical points of $I$ give classical $T$-periodic solutions of (HS) (see [9]). We set

$$
I^{\varepsilon}=\{q \in \Lambda: I(q)<\varepsilon\} .
$$

4.7. Proposition. Assuming (VI)-(V3), there is an $\varepsilon>0$ and an $R>0$ such that if $B(0, R)$ is the open ball of radius $R, A=\mathbb{R}^{n}-B(0, R), B=\Lambda-I^{\varepsilon}$, then

1. $\sup _{A} I<\varepsilon$

2. $I^{\varepsilon}$ is deformable into $A$.

3. $\operatorname{cat}_{\Lambda_{T}}(E-B, A)=0$, $\operatorname{cat}_{\Lambda_{T}}(\Lambda, A)=+\infty$.

Proof. First we choose a decreasing sequence $\varepsilon_{m}>0$ such that $\varepsilon_{m} \rightarrow 0$ and a corresponding increasing sequence $R_{m}>0$ such that $R_{m} \rightarrow+\infty$ with the property that

$$
|V(t, q)|<\varepsilon_{m} \text { implies }|q|>R_{m} .
$$

Choose an index $k$ such that

$$
R_{k}-\left[2 \varepsilon_{k}\right]^{\frac{1}{2}} T>\left[2 \varepsilon_{k}\right]^{\frac{1}{2}} T
$$

and

$$
-\int_{0}^{T} V(t, q) d t<\frac{1}{2} \varepsilon_{k} T, \quad \text { for }|q| \geq R_{k}
$$

and set $\varepsilon=T e_{k}, R=R_{k}, A=\mathbf{R}^{n}-B(0, R), B=\Lambda-I^{\varepsilon}$. If $q \in I^{\varepsilon}$,

$$
\|\dot{q}\|_{L_{2}}^{2}=\int_{J_{0}}^{T}|\dot{q}|^{2} \leq 2 I(q) \leq 2 T \varepsilon_{k}
$$


and hence

$$
\|\dot{q}\|_{L_{2}} \leq(2 \varepsilon)^{\frac{1}{2}} \text {. }
$$

Now, write $q=[q]+Q$, where $Q(t)=q(t)=[q]$ and

$$
[q]=\frac{1}{T} \int_{0}^{T} q(t) d t \text {. }
$$

Recall that

$$
\|Q\|_{L_{\infty}}=\max _{t}|Q(t)|
$$

and the general inequality [9],

$$
\|Q\|_{\infty} \leq T^{\frac{1}{2}}\|\dot{q}\|_{L_{2}}
$$

Hence,

$$
\|Q\|_{\infty} \leq(2 T \varepsilon)^{\frac{1}{2}}
$$

Now, consider a constant loop $q$, with $|q| \geq R$.

$$
I(q)=-\int_{0}^{T} V(t, q) d t<\frac{\varepsilon}{2}<\varepsilon
$$

and hence $A=\mathbf{R}^{n}-B(0, R) \subset I^{\varepsilon}$ and

$$
\sup _{A} I<\varepsilon \text {. }
$$

Consider now the homotopy, $H: I^{\varepsilon} \times[0,1] \rightarrow \Lambda$, where

$$
H(q, s)=[q]+(1-s) Q, \quad 0 \leq s \leq 1
$$

which is fixed on constant loops $q$. For $q \in I^{\varepsilon}$, it is easy to verify that

$$
|[q]| \geq R-(2 T \varepsilon)^{\frac{1}{2}}>(2 \varepsilon T)^{\frac{1}{2}}
$$

and using (8)

$$
|Q(t)| \leq(2 T \varepsilon)^{\frac{1}{2}} \text { for all } t \in[0,1]
$$

This forces

$$
[q]+(1-s) Q(t) \neq 0, \quad 0 \leq s, \quad t \leq 1 .
$$

Thus the homotopy has range in $\Lambda$ and deforms $I^{\varepsilon}$ into the subspace $\mathbb{R}^{n}-$ $B(0, \rho), \rho=(2 \varepsilon T)^{1 / 2}$. If one follows $H$ by a radial homotopy, we obtain a deformation of $I^{\varepsilon}$ to $\mathbf{R}^{n}-B(0, R)$, with $\mathbf{R}_{n}-B(0, R)$ fixed throughout the composite homotopy. This also shows that cat $_{\Lambda}(\Lambda-B, A)=0$. Finally, since $\operatorname{cat}_{\Lambda} A=2$ and cat $_{\Lambda}=+\infty$, it is clear that $\operatorname{cat}_{\Lambda}(\Lambda, A)=+\infty$.

4.8. Theorem. (Rabinowitz[9]). If we assume that $\mathrm{V}$ satisfies (V1)-(V4), the function I possesses an unbounded sequence of critical values.

Proof. Rabinowitz [9] verifies that $I$ satisfies (PS) for all $s>0$ and we will not repeat the argument. In view of Proposition 4.7, the proof is now a direct application of Theorem 4.6 . 


\section{REFERENCES}

1. A. Ambrosetti and V. Coti Zelati, Critical points with lack of compactness and singular dynamical systems, Ann. Mat. Pura Appl. (to appear).

2. E. Fadell, On fiber spaces, Trans. Amer. Math. Soc. 90 (1959), 1-14.

3. __ Cohomological methods in non-free G-spaces with applications to general Borsuk-Ulam theorems and critical point theorems for invariant functionals, Nonlinear Functional Analysis and its Applications, Reidel, (1986), 1-47.

4. __ Lectures in cohomological index theories of G-spaces with applications to critical point theory, Raccolta Di Universeta della Calabria, (1987), Cosenza, Italy.

5. E. Fadell and S. Husseini, Relative cohomological index theories, Adv. in Math. 64 (1987), $1-31$.

6. E. Fadell and L. Neuwirth, Configuration spaces, Math. Scand. 10 (1962), 111-118.

7. W. Hurewicz, On the concept of fiber space, Proc. Nat. Acad. Sci. 41 USA (1955), 956-961,

8. P. Rabinowitz, Minimax methods in critical point theory with applications to differential equations, CBMS Report No. 65, 1984.

9. ___ Periodic solutions for some forced singular Hamiltonian systems, Festschrift fur Jurgen Moser (to appear).

10. J. T. Schwartz, Generalizing the Lusternik-Schnirelmann theory of critical points, Comm. Pure. Appl. Math. 17 (1964), 307-315.

11. J. P. Serre, Homologie singulier des espaces fibrés, Ann. of Math. 54 (1951), 425-505.

12. E. Spanier, Algebraic topology, McGraw-Hill, 1966.

13. M. Viguré-Poirrier and D. Sullivan, The homology theory of the closed geodesic problem, J. Differential Geom. 11 (1976), 633-644.

Department of Mathematics, The University of Wisconsin-Madison, Madison, WISCONSIN 53706 